
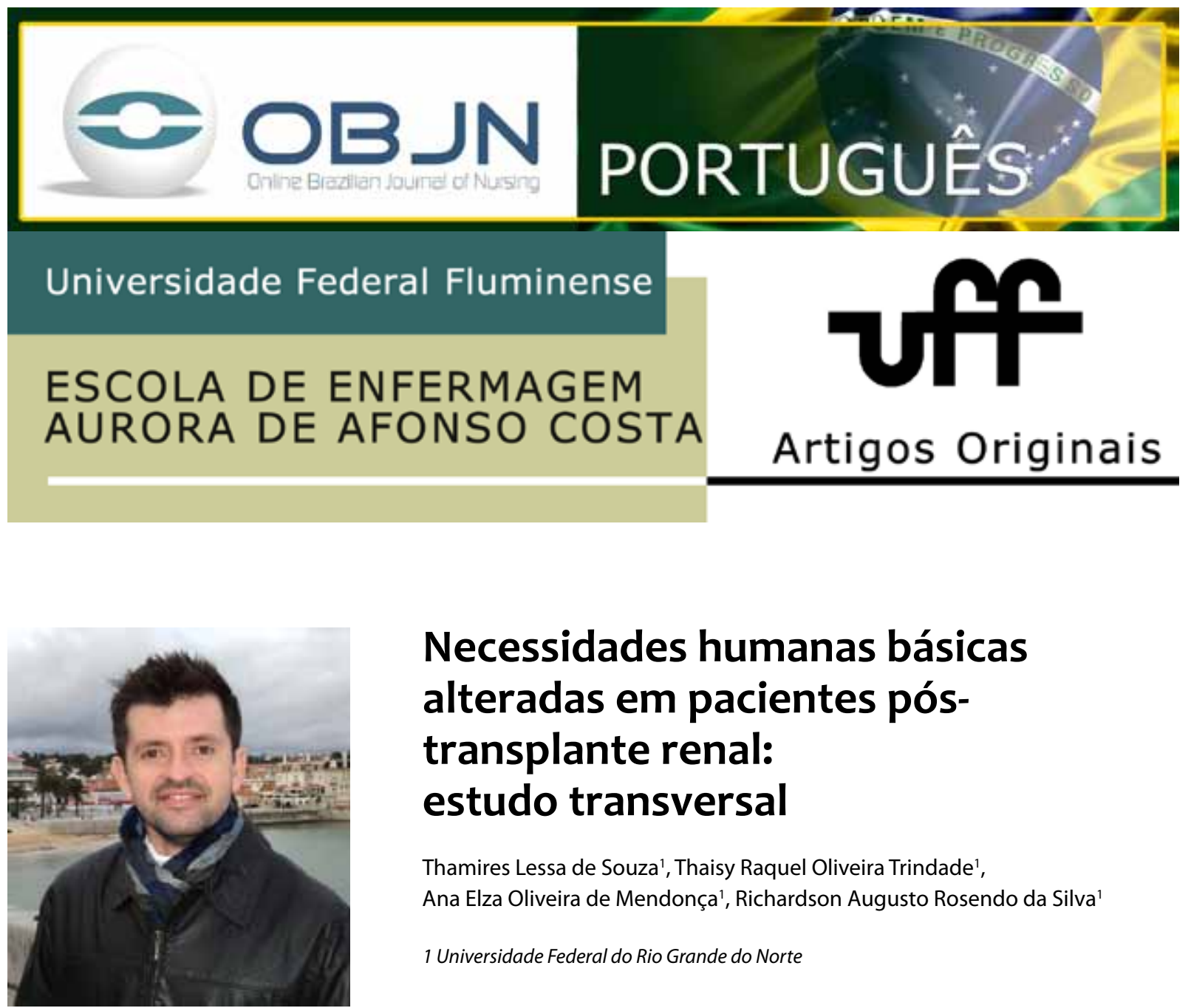

\title{
Necessidades humanas básicas alteradas em pacientes pós- transplante renal: estudo transversal
}

Thamires Lessa de Souza', Thaisy Raquel Oliveira Trindade',

Ana Elza Oliveira de Mendonça' ${ }^{1}$ Richardson Augusto Rosendo da Silva'

1 Universidade Federal do Rio Grande do Norte

\section{RESUMO}

Objetivo: identificar as necessidades humanas básicas alteradas nos pacientes que realizaram transplante renal, à luz da Teoria das Necessidades Humanas Básicas. Método: estudo transversal de abordagem quantitativa, realizado com 68 pacientes em pós-operatório de transplante renal em um hospital do Nordeste brasileiro entre os meses de outubro de 2013 e março de 2014. O instrumento de coleta de dados utilizado foi um roteiro de anamnese e exame físico. Os resultados foram analisados por meio da Teoria de Horta. Resultados: foram identificadas 13 necessidades alteradas, sendo as mais frequentas: eliminação, oxigenação, hidratação, integridade cutâneo-mucosa, sono/repouso, nutrição e lazer. Conclusão: o estudo permitiu identificar que as necessidades humanas básicas alteradas nos pacientes que realizaram transplante renal, estavam divididas em Psicobiológicas e Psicossociais, sendo relevantes para subsidiar a elaboração de um plano de cuidados mais específico para essa clientela.

Descritores: Teoria de enfermagem; Processos de Enfermagem; Cuidados de Enfermagem; Transplante de Rim. 


\section{INTRODUÇÃO}

A insuficiência renal crônica (IRC) é uma doença que pode afetar todo o organismo, culminada por diversas afecções renais ou do trato urinário(1). É resultado da diminuição gradual ou da perda irreversível das funções renais, caracterizada por redução da taxa de filtração glomerular e/ou lesões no parênquima renal em um período igual ou superior a três meses ${ }^{(2-3)}$.

Atualmente, a IRC é um problema de saúde mundial e no Brasil atinge mais de 90 mil pessoas, com maior incidência na faixa etária de 19 a 64 anos. Esses dados tendem a crescer devido ao aumento das doenças crônicas, envelhecimento populacional e demora no diagnóstico dessa doença(4).

O tratamento para IRC consiste em associação entre terapia farmacológica, nutrição equilibrada, diálise e, quando possível, o transplante renal. A escolha da modalidade do tratamento vai depender de alguns fatores, como doença de base, estágio da doença, velocidade da diminuição da filtração glomerular e comorbidades associadas $^{(2,5)}$.

O transplante renal proporciona melhor qualidade de vida para os pacientes com IRC, porém o acesso não ocorre de forma simples, já que a demanda é crescente e o número de rins disponíveis não acompanha seu aumento. Além disso, envolve também a questão de se encontrar um doador compatível com o paciente e, por fim, o risco de haver rejeição do órgão, trazendo esse paciente à condição inicial de disfunção renal( ${ }^{(6)}$.

É importante ressaltar que a pessoa com IRC em tratamento dialítico, especialmente hemodiálise, apresenta várias necessidades: conviver com um acesso para o tratamento representado pela fístula arteriovenosa, enxerto ou cateter duplo lúmen, restrições alimentares e volumes de líquidos. Essa realidade traz im- pactos negativos para o ser biopsicossocial, bem como no estilo de vida da pessoa e sua família ${ }^{(2,3)}$.

Considerando a complexidade do procedimento cirúrgico, o paciente pós-transplante renal apresenta inúmeras necessidades, quais sejam: suporte emocional e psicoterápico para adaptação ao novo órgão e o preparo para reestruturação da vida sócio-familiar e sociolaborativa pós-cirurgia; necessidades sociais para adequar-se ao novo ritmo de vida após a alta hospitalar e econômicas para arcar com as despesas inerentes à sua nova condição de vida ${ }^{(5,6)}$.

Ademais, o paciente pós-transplante renal também necessita de uma assistência à saúde direta e continua, devido à importância de monitorização dos parâmetros da função hepática, renal, hematológica, bioquímica do sangue, o rastreamento rigoroso de possíveis sinais de infecção, o uso contínuo de medicamentos e os ajustes do esquema imunossupressor ${ }^{(5,6)}$.

Nesse sentido, o enfermeiro pode fornecer uma assistência integralizada ${ }^{(7)}$ fundamentando-se no processo de enfermagem associado a uma teoria, pois confere mais segurança e qualidade à assistência do profissional ${ }^{(8)}$.

Para a implementação do cuidado de enfermagem, o enfermeiro dispõe da Sistematização da Assistência de Enfermagem (SAE), a qual pode ser norteada pelo Processo de Enfermagem (PE). Ela organiza o trabalho profissional quanto ao método, pessoal e instrumentos. Dessa forma, a SAE pode auxiliar o enfermeiro a organizar o seu trabalho, diminuindo o risco de rejeição do órgão transplantado e aumentando a qualidade dos serviços prestados ${ }^{(9)}$.

As teorias de enfermagem surgiram para fundamentar a profissão como ciência e promover qualificação profissional. O PE, quando baseado em uma teoria, garante mais segurança na assistência do enfermeiro, além de respaldo e caráter científico ${ }^{(3)}$. 
O PE foi introduzido no Brasil por Wanda de Aguiar Horta por meio de um modelo teórico denominado Teoria das Necessidades Humanas Básicas. Seu modelo abrange leis que regem os fenômenos naturais: a lei do equilíbrio (homeostase), a lei da adaptação e a lei do holismo ${ }^{(10)}$.

A teoria de Horta baseia-se na manifestação das necessidades humanas básicas, que são compreendidas como estados de tensão essenciais à sobrevivência e resultantes de desequilíbrios homeostáticos. Elas são vitais, flexíveis, cíclicas, dinâmicas, inter-relacionadas e universais. São comuns a todos os humanos, no entanto a forma como elas se mostram e como são solucionadas é o que vai individualizar cada ser ${ }^{(10)}$.

As necessidades são divididas em psicobiológicas, que são instintivas, como oxigenação e nutrição; em psicossociais, caracterizadas como aquelas que são geradas a nível social, como recreação e comunicação; e as psicoespirituais, aquelas em que o homem procura interpretar o que cientificamente não tem explicação(8).

Diante do panorama apresentado e para justificar o desenvolvimento do estudo, buscou-se por produções científicas sobre Necessidades humanas básicas alteradas em pacientes pós-transplante renal utilizando os descritores teoria de enfermagem; processos de enfermagem; cuidados de enfermagem e transplante de rim, adotando como limite temporal os últimos cinco anos nas bases de dados Literatura Latino-Americana e do Caribe (Lilacs), Literatura Internacional em Ciências da Saúde e Biomédica (Medline), Base de dados em Enfermagem (BDENF), SCOPUS, Web of Science e CINAHL. Verificou-se escassez de estudos relacionados à referida temática, sobretudo de pesquisas atuais, justificando a realização do presente estudo.

Além disso, esta pesquisa é relevante pela contribuição que o enfermeiro pode fornecer na identificação das necessidades humanas básicas em desequilíbrio, como elemento para conduzir a elaboração de um plano de ações o mais precoce possível que direcione o tratamento e atendimento das necessidades do paciente, visando qualificar a assistência de enfermagem ao paciente no pós-operatório. Atentando para fatores de risco que podem levar a possíveis complicações, como também guiar o desenvolvimento de futuras investigações, contribuindo, dessa forma, para a construção do conhecimento e engrandecimento da enfermagem.

A partir destes pressupostos no estudo em tela emergiu o seguinte questionamento: quais as necessidades humanas básicas alteradas com o desequilíbrio nos pacientes que se submeteram ao transplante renal?

Desse modo, este estudo teve como objetivo identificar as necessidades humanas básicas alteradas nos pacientes que se submeteram ao transplante renal, à luz da Teoria das Necessidades Humanas Básicas.

\section{MÉTODO}

Trata-se de um estudo transversal, com abordagem quantitativa, realizado em um hospital universitário mantido pelo Sistema Único de Saúde, situado no Rio Grande do Norte. A escolha do local se deu por ser referência em transplante renal na referida região e credenciado na Associação Brasileira de Transplante de Órgãos (ABTO).

A população foi composta de 82 pacientes transplantados renais, a qual representa a média aritmética dos transplantes realizados nos últimos cinco anos (2008 a 2012) no referido hospital, extraídos do sistema de cadastramento.

Calculou-se o tamanho da amostra a partir da fórmula para populações finitas, levando em consideração o nível de confiança de 95\% $(Z \infty=1,96)$, o erro amostral de $5 \%$, o tamanho 
da população e a prevalência de transplantes renais no $\mathrm{RN}$ encontrada de $0,02 \%{ }^{(4)}$. Entretanto, para estimativa do tamanho da amostra, considerou-se a prevalência de $1 \%$ com o intuito de aumentar seu tamanho. Assim, a amostra foi constituída por 68 pacientes. A seleção dos pacientes foi obtida por meio da amostragem por conveniência do tipo consecutiva. A pesquisa se realizou entre os meses de outubro de 2013 e março de 2014. Os critérios de inclusão para os participantes do estudo foram: serem maiores de 18 anos; transplantados no serviço; em pós-operatório mediato; acompanhados no hospital e estarem em condições físicas adequadas para a realização da anamnese e exame físico. Excluíram-se pacientes que não estavam em condições físicas e mentais adequadas para a realização da anamnese e/ou exame físico.

Respeitaram-se os aspectos éticos em pesquisa, como participação voluntária dos pacientes e assinatura prévia do Termo de Consentimento Livre e Esclarecido.

O instrumento utilizado na coleta de dados foi um roteiro de anamnese e exame físico, baseado na Teoria das Necessidades Humanas Básicas, o qual incluía dados socioeconômicos e do transplante, queixa principal, independência funcional e neurológica, nutrição, eliminação, sono/repouso/atividade, relacionamentos, tolerância ao estresse, sentimentos de fé, segurança/proteção e conforto, além de exame físico detalhado.

O instrumento foi submetido à validação do conteúdo e aparência por enfermeiros docentes, entre os meses de agosto e setembro de 2013. A amostra foi selecionada de forma intencional a partir da avaliação de seus currículos. Para tanto, realizou-se uma busca por meio da plataforma Lattes, do Conselho Nacional de Desenvolvimento Científico e Tecnológico (CNPq).

Os critérios de inclusão foram: ser docente do curso de Graduação em Enfermagem, ter desenvolvido estudo publicado ou de conclusão de titulação (especialização, mestrado ou doutorado) relacionado à Sistematização da Assistência de Enfermagem, teorias de enfermagem e transplante renal, ter orientações acadêmicas na área; já como critério de exclusão, informar no Currículo Lattes apenas o Trabalho de Conclusão do Curso de Graduação sobre a temática. O resultado foi de 22 docentes.

Após a seleção dos professores, foi enviada uma carta convite via correio eletrônico, esclarecendo os objetivos da pesquisa e o termo de consentimento livre e esclarecido. Assim, após a confirmação do aceite em participar da pesquisa e a assinatura no termo de consentimento livre e esclarecido, foi enviado o instrumento para análise.

Dos 22 convites enviados para validação do instrumento, obteve-se o retorno de 14 respostas, sendo quatro de negação e 10 de aceite. Assim, para o seguimento desta etapa, foram encaminhados para os 10 docentes que aceitaram o convite em participarem da pesquisa o instrumento e as instruções de preenchimento deste via e-mail.

As sugestões propostas foram contempladas no instrumento, posteriormente aplicado, sob a forma de pré-teste, a dez pacientes submetidos à diálise peritoneal. Não houve necessidade de alterações, com os participantes do pré-teste sendo incluídos na amostra do estudo. Ressalta-se que o mesmo foi aplicado pelos pesquisadores individualmente com cada paciente no dia da consulta ambulatorial, em uma sala reservada, livre de interrupções e de forma a preservar a privacidade destes.

Os dados coletados foram analisados a partir da Teoria de Wanda de Aguiar Horta para identificar as necessidades humanas alteradas. Ressalta-se que no processo de identificação das referidas necessidades alteradas, as histórias clínicas foram individualmente avaliadas por dois 
autores deste artigo, sendo os dois doutores, a fim de possibilitar maior fidedignidade aos resultados obtidos. Os resultados que apresentaram concordância entre estes foram aceitos. Aqueles em que havia discordância entre os avaliadores, eram reavaliados em suas histórias clínicas até que se obtivesse um consenso. Além disso, os dados foram transferidos para o programa Microsoft Excel ${ }^{\circledR}$ para a realização da estatística descritiva e organizados em tabelas com frequências absolutas e percentuais.

A pesquisa se desenvolveu respeitando os aspectos éticos regulados pelas Diretrizes e Normas de Pesquisa em Seres Humanos, Resolução 466/12 do Conselho Nacional de Saúde, e foi aprovada pelo Comitê de Ética em Pesquisa (CEP) do hospital universitário para iniciar a coleta de dados, com o Parecer de número 398.678, data de aprovação 18/09/2013 e CAAE 19473613.2.0000.5537. Além disso, a todos os participantes foram explicados os objetivos da pesquisa, lido o Termo de Consentimento Livre e Esclarecido e solicitada a assinatura deles.

\section{RESULTADOS}

Os pacientes eram, em sua maioria, do sexo masculino (57,15\%), na faixa etária de 18 a 65 anos, com média de 30-40 anos. Em relação ao estado civil, $57,15 \%$ eram casados, $28,6 \%$, solteiros e $14,25 \%$ em união estável. Dentre os entrevistados, 64,3\% eram aposentados ou estavam em auxílio doença pelo Instituto Nacional do Seguro Social (INSS), já o restante consideraram-se do lar.

Os usuários tinham naturalidade do interior do Rio Grande do Norte, num total de $50 \%$, já os outros $50 \%$ estavam divididos entre a capital do RN $(35,7 \%)$ e outros estados do Brasil (14,3\%). A religião predominante foi a católica, com $71,5 \%$ dos entrevistados, porém todos apresentavam o sentimento da fé. O tempo de espera para a realização do transplante, em $42,9 \%$, foi entre 6-10 anos; $90 \%$ receberam órgão de doador falecido e $10 \%$, de doador vivo.

Foram identificadas 13 necessidades humanas básicas alteradas, as quais estavam relacionadas apenas as necessidades psicobiológicas e psicossociais. Entretanto, somente sete estavam presentes em pelo menos $50 \%$ da amostra. São elas: Eliminação, Oxigenação, Integridade cutâneo-mucosa, Nutrição, Hidratação, Sono e Repouso e Lazer.

A Tabela 1 apresenta a distribuição das Necessidades Humanas Básicas alteradas nos pacientes pós-transplante renal, divididas em necessidades psicobiológicas e psicossociais.

Tabela 1 - Necessidades Humanas Básicas divididas em necessidades psicobiológicas e psicossociais em pacientes pós-transplante renal, Natal, 2014.

\begin{tabular}{lccccc}
\hline $\begin{array}{l}\text { Necessida- } \\
\text { des Psico- } \\
\text { biológicas }\end{array}$ & N & $\%$ & $\begin{array}{c}\text { Neces- } \\
\text { sidades } \\
\text { Psicos- }\end{array}$ & N & $\%$ \\
\hline Eliminação & 68 & 100 & Lazer & 55 & 80,8 \\
$\begin{array}{l}\text { Integridade } \\
\text { Cutâneo- }\end{array}$ & 59 & 86,7 & Recreação & 28 & 41,1 \\
-Mucosa & & & & & \\
$\begin{array}{l}\text { Nutrição } \\
\text { Sono e }\end{array}$ & 55 & 80,8 & $\begin{array}{c}\text { Comuni- } \\
\text { cação }\end{array}$ & 14 & 20,5 \\
$\begin{array}{l}\text { Repouso } \\
\text { Hidratação }\end{array}$ & 37 & 60,2 & Aceitação & 12 & 17,6 \\
$\begin{array}{l}\text { Oxigenação } \\
\text { Exercício e }\end{array}$ & 36 & 52,9 & Liberdade & 5 & 7,3 \\
$\begin{array}{l}\text { Atividades } \\
\text { Físicas }\end{array}$ & 31 & 45,5 & & & \\
$\begin{array}{l}\text { Cuidado } \\
\text { Corporal }\end{array}$ & 14 & 20,5 & & & \\
\hline Fonte:autoriapropria, & & & & & \\
\hline
\end{tabular}

Fonte: autoria própria, 2014.

\section{DISCUSSÃO}

Após a coleta dos dados, foi observado que os achados relacionados ao perfil socio- 
demográfico dos pacientes, aproximam-se de outros estudos com pacientes que já realizaram transplante renal ${ }^{(7,9,11,12)}$.

Em um estudo com 835 pacientes de Belo Horizonte (MG), os homens eram predominantes nos casos de pacientes que necessitavam realizar transplante renal, como analisado nesta pesquisa ${ }^{(7)}$. Já, em relação à idade, outra pesquisa com 98 prontuários mostrou que a faixa etária média foi 51 anos, contradizendo esta pesquisa, que apresentou uma faixa etária entre 30-40 anos $^{(11)}$.

Em outro estudo, também realizado em um hospital universitário, foi identificado que a maioria dos pacientes com IRC eram aposentados e apresentavam companheiro, além de seguirem alguma religião ${ }^{(12)}$. Confirmando o que foi observado nesta pesquisa.

Em relação ao tempo de espera para a realização do transplante renal, foi observado, em um estudo realizado na cidade de Fortaleza/CE que o tempo médio foi de 6,50 anos ${ }^{(9)}$. Aproximando-se dos achados desta pesquisa, que teve média de 6 a 10 anos.

Este longo tempo de espera para o transplante pode ser justificado pelo sistema de saúde do Brasil apresentar algumas dificuldades, como a não aceitação da família para doação dos órgãos de seus parentes; a falta de assistência social às famílias dos pacientes que entraram em protocolo de morte encefálica, assim como dificuldade na notificação desses casos; dificuldades na captação dos órgãos e distribuição entre os estados ${ }^{(5,6,13)}$.

Dentre as necessidades humanas básicas alteradas, a eliminação foi a mais frequente, estando presente em todos os participantes do presente estudo, os quais apresentaram a poliúria como sinal de desequilíbrio. Esta Necessidade Psicobiológica, é muito comum no pós-transplante renal, pela modificação na produção urinária relacionado à presença do rim transplantado e da fase de adaptação, na qual o organismo se encontra ${ }^{(14)}$.

Nesse sentido o enfermeiro deve monitorar a eliminação urinária, inclusive frequência, consistência, odor, volume e cor; anotar o horário da última eliminação de urina e orientar o paciente/ família a registrar o débito urinário, conforme apropriado e avaliar o estado de hidratação do paciente $\mathrm{e}^{(7,9)}$.

Em relação à Integridade Cutâneo-Mucosa, classificada como necessidade psicobiológica, foi constatado que todos os pacientes que realizaram transplante renal utilizavam drogas imunossupressoras durante o tratamento, como o corticosteroide prednisona, o qual pode promover alterações na pele, tais como: petéquias, retardo na cicatrização, além de outros efeitos dermatológicos. Além da afecção em si já promover mais sensibilidade da pele a escoriações e à presença da ferida operatória. Essa necessidade também foi notada, pois a maioria dos pacientes fazia uso de medicações intravenosas e apresentava descontinuidade da pele pelo uso de cateteres ${ }^{(15)}$.

É importante destacar que os métodos invasivos, como a diérese (cirurgia dos tecidos orgânicos), são situações de risco para colonização de bactérias, ao qual pode afetar a estrutura imunológica do paciente. Por isso, o enfermeiro deve ter habilidade e competência para implementar intervenções, como a avaliação diária do curativo, e a sua troca; fazer, orientar, auxiliar a mudança de decúbito e manter o paciente limpo e seco.

Diante dessa necessidade alterada o paciente está vulnerável ao risco de infecção ${ }^{(7,9)}$.

Assim, o enfermeiro deve estabelecer como meta de cuidado, manter o estado imunológico adequado do paciente, ou seja, a resistência natural e adquirida por meio das seguintes intervenções: avaliação da saúde, identificação de risco e utilização de medidas de precaução universal ${ }^{(2,3)}$. 
A Nutrição também merece destaque, pois muitos pacientes apresentavam essa necessidade psicobiológica alterada devido à êmese, que poderia culminar em emagrecimento e déficit no sistema imunológico. Uma possível causa para esse quadro é o acúmulo de ureia na corrente sanguínea, a qual pode se difundir para o trato gastrointestinal e causar náuseas e êmese ${ }^{(3)}$.

Outro fator para essa sintomatologia é a hipercalcemia, visto que o nefropata acumula cálcio na corrente sanguínea que, em excesso, estimula a secreção de ácido clorídrico pelo estômago, deixando o organismo mais sensível a náuseas e vômitos ${ }^{(16)}$.

Geralmente, os pacientes pós-transplante renal podem apresentar desequilíbrio nutricional por excesso, porque a alimentação passa a ser menos restrita do que na terapia dialítica e o apetite é aumentado pelo uso de drogas imunossupressoras $^{(9)}$.

Dessa forma, o enfermeiro deve promover o estado nutricional dos pacientes por meio das seguintes atividades: determinar a ingestão alimentar e os hábitos alimentares do pós-transplante renal; discutir sobre as exigências nutricionais e as percepções destes quanto à dieta prescrita\recomendada; e providenciar encaminhamentos\consultas com outros membros da equipe de saúde conforme apropriado; pesar o paciente a intervalos específicos; monitorar as tendências de aumento e perda de peso; monitorar os níveis de albumina, proteína total, hemoglobina e hematócritos, linfócitos e eletrólitos; monitorar o nível de energia, mal estar, cansaço e fraqueza ${ }^{(2,3,7,9)}$.

Outra necessidade psicobiológica contemplada no estudo foi a Oxigenação. O paciente renal crônico e pós-transplantado mediato apresenta dispneia e fadiga constantemente devido, muitas vezes, à anemia presente. Mas não necessariamente a anemia é causada por esse motivo. Esta complicação se inicia pelo déficit na produção de eritropoetina pelos rins, pois com a redução da função renal diminui o estímulo para a eritropoese na medula óssea ${ }^{(16)}$.

Ressalta-se que a fadiga pode contribui para a restrição do paciente ao leito e, com a deambulação prejudicada, a rede venosa não funciona de forma correta, aumentando o risco de úlcera por pressão, embolia pulmonar e trombose venosa profunda ${ }^{(17,18)}$.

Diante disso, o enfermeiro deve realizar o controle da energia por meio da suplementação nutricional por folato associado à cianocobalamina para que possa estimular a produção de eritropoietina, melhorando a sua condição física e de capacidade funcional Entretanto, a eritropoetina em excesso pode trazer alguns efeitos colaterais, como hipertensão e complicações trombóticas ${ }^{(15)}$.

Além disso, o enfermeiro pode estabelecer como metas do plano de cuidados manter a tolerância a atividade dos pacientes. Nesse sentido, pode estimular a promoção da prática de exercícios ativos e passivos ${ }^{(17,18)}$.

A Hidratação também foi uma necessidade psicobiológica com grandes alterações nos pacientes renais crônicos e em pós-operatório de transplante. Sem a função renal plena, o indivíduo tende a reter sódio, água, acumular substâncias no sangue, culminando em sobrecarga hídrica e ocasionando em edema e hipertensão(13).

Nesse aspecto, o enfermeiro deve estabelecer como meta o equilíbrio hídrico e assim, realizar as intervenções necessárias como o controle hídrico, controle hidroeletrolítico, monitoração hídrica, avaliação da presença de edema e realização do balanço hídrico(17,18).

A necessidade psicobiológica Sono e Repouso, também foram identificados na pesquisa a qual é fundamental ser observada, pois, quando em desequilíbrio, torna o indivíduo irritado e cansado(9). O período de internação 
e a própria afecção tornam os pacientes mais susceptíveis a distúrbios nessa temática, os quais ficam preocupados com questões financeiras por estarem fora de casa e surge o medo de rejeição do enxerto ${ }^{(9)}$.

Além disso, em virtude do aumento da ingestão hídrica e do uso de diuréticos após o transplante, o transplantado interrompa o sono várias vezes durante a noite, em virtude da urgência urinária, o que contribui para o aparecimento dessa necessidade alterada ${ }^{(19,20)}$.

Diante dessa necessidade alterada, o enfermeiro visando promover o sono e repouso adequado do paciente pode realizar intervenções como: ensinar ao cliente técnicas de relaxamento; determinar o efeito dos medicamentos sobre o sono; discutir com o paciente e a família as medidas de conforto, técnicas de monitoramento do sono e as mudanças no estilo de vida e oferecer ambiente calmo e agradável para o descanso adequado ${ }^{(17,18)}$.

Por fim, a última necessidade identificada, classificada como psicossocial foi o Lazer, a qual é foi fundamental para o bem-estar e qualidade de vida dos pacientes. O período de hospitalização é desagradável, pois determina algumas mudanças nos hábitos diários dos pacientes. Algumas atividades de lazer e recreação podem promover mais conforto para os clientes, assim como favorecer a manutenção de relacionamento interpessoal e afastamento da solidão(17).

Dessa forma, o enfermeiro deve desenvolver atividades relaxantes e de lazer compatíveis com a sua condição clínica do paciente, bem como rever a história de atividades/passatempos prediletos e possíveis modificações ${ }^{(19,20)}$.

As necessidades humanas básicas alteradas listadas anteriormente são relevantes, visto que englobam quase todos os pacientes renais crônicos no pós-transplante renal. Estes desenvolvem essas necessidades pela própria fisiopatologia da doença renal e à enfermagem cabe planejar e implementar precocemente ações que minimizem-nas ${ }^{(19)}$. Nesse sentido, a utilização do referencial Teoria de Horta pode proporciona ao enfermeiro meios para organizar as informações e os dados dos clientes, para analisar e interpretar esses dados e avaliar os resultados do processo de cuidar. Assim, permiti instrumentalizar o planejamento científico e sistematizar as ações desenvolvidas pelo enfermeiro, dando maior visibilidade a enfermagem enquanto ciência ${ }^{(20)}$.

Tais necessidades são importantes de serem observadas porque graças a elas o enfermeiro seleciona quais pacientes apresentam maiores riscos de desenvolver complicações, como o risco de rejeição do enxerto e hipertensão maligna ${ }^{(18)}$. Além de evitar problemas futuros e garantir um atendimento de qualidade.

Um estudo demostrou que a promoção de uma linha de cuidado crônico intenso, liderada pelo enfermeiro, poderia trazer inúmeros benefícios para o paciente, por exemplo, redução de atendimentos de urgência e internações, causados por desequilíbrios nas necessidades básicas. A intervenção incluiu decisão compartilhada e reorganização do sistema de saúde ${ }^{(19)}$.

Destarte, a enfermagem pode atuar de maneira expressiva almejando sua atuação baseada na identificação de respostas humanas e no estabelecimento de estratégias que proporcionem a recuperação da saúde ou a melhoria do bem-estar individual ou coletivo ${ }^{(2,5)}$.

O cuidado de enfermagem é importante desde o período pré até o pós-transplante, pois as necessidades básicas podem entrar em desequilíbrio em qualquer fase do processo de adoecimento e pré-reabilitação. Durante a fase pré-operatória, a enfermagem atua no preparo das condições biológicas e, principalmente, das emocionais, por exemplo, esclarecendo dúvidas sobre o procedimento cirúrgico, a fim de reduzir a ansiedade do paciente e família ${ }^{(20)}$. No período 
intraoperatório, o enfermeiro atua, junto à equipe multiprofissional, tentando reduzir possíveis complicações no ato cirúrgico e realizando procedimentos próprios desse período, como sondagem vesical ${ }^{(20)}$.

Já, em relação ao pós-operatório, o enfermeiro age avaliando e intervindo antecipadamente nas possíveis complicações ${ }^{(20)}$. Nesse período é muito comum surgirem desequilíbrios das necessidades, como distúrbios na eliminação, além de oscilações na pressão arterial, alteração no sono, nas condições hidroeletrolíticas, na nutrição e risco de infecção ${ }^{(9,18,20)}$.

$O$ paciente que se encontra internado merece atenção especial durante toda a fase do seu tratamento, visto que apresenta suas necessidades humanas básicas alteradas e essa condição pode levá-lo a uma situação de risco e prolongamento do seu estado de doença. Por isso, é imprescindível assistência de enfermagem qualificada, imediata e contínua.

\section{CONCLUSÃO}

A pesquisa em questão proporcionou a identificação de 13 necessidades humanas básicas alteradas: eliminação, comunicação, oxigenação, lazer, integridade cutâneo-mucosa, recreação, cuidado corporal, liberdade, nutrição, aceitação, hidratação, sono e repouso, exercício e atividades físicas. Entretanto, apenas sete foram discutidas por serem predominantes na amostra estudada.

A identificação das necessidades humanas básicas alteradas nos pacientes pós-transplantados renais, dá subsídios ao enfermeiro, para a elaboração de um plano de cuidados específico para essa clientela. Além disso, a partir do conhecimento de tais respostas humanas, torna-se possível predizer, prever, detectar e controlaras complicações potenciais.
Diante das complicações potenciais existentes na vida desses indivíduos, que podem comprometer a sobrevida do enxerto renal e do próprio paciente, percebe-se a necessidade de pensar a prática assistencial direcionada na identificação das necessidades humanas básicas alteradas nesses pacientes, que sirvam de parâmetro para traçar metas e definição de intervenções específicas para essa clientela.

Dessa forma, a utilização do modelo teórico de Horta poderá subsidiar a sistematização da assistência de Enfermagem aos pacientes pós-transplantados renais, por contribuir para a organização do trabalho do enfermeiro, direcionar as intervenções para redução do risco de rejeição, o aumento da qualidade de vida desses usuários e a visibilidade da enfermagem enquanto ciência.

Por fim, os limites do estudo estiveram relacionados ao tipo de amostragem não probabilística empregada no estudo, na qual o pesquisador seleciona os elementos a que tem acesso, o que não garante a representatividade da amostra, dificultando a generalização dos resultados. Entretanto, espera-se que os resultados do estudo contribuam para a padronização da linguagem específica de enfermagem e estimule outras pesquisas na área. Desse modo, considera-se que outros estudos devam ser desenvolvidos com o intuito de comparar os dados de realidades diferentes.

\section{REFERÊNCIAS}

1. Araújo AM, Mendonça AEO de, Rodrigues MP, Torres GV. Identifying risk factors for chronic renal insufficiency in the group friends of the heart. Rev enferm UFPE on line[internet]. 2012[Cited 2015 Apr 15]; 6(3): 578-86. Available from: http://www. revista.ufpe.br/revistaenfermagem/index.php/ revista/article/viewArticle /2311 
2. Reddenna L, Basha SA, Reddy KSK. Dialysis Treatment: a comprehensive description. Int J of Pharm Res \& All Sci[internet]. 2014[Cited 2015 Apr 5];3(1):1-13.Available from: http://ijpras.com/ volume-3-issue-1/IJPRAS-2014-3-1-1-13.pdf

3. Lins SMSB, Santo FHE, Fuly PSC, Garcia TR. Subset of ICNP ${ }^{\circledR}$ diagnostic concepts for patients with chronic kidney disease. Rev bras Enferm [internet]. 2013[Cited 2015 May 5]; 66(2): 180-9. Available from:http://www.scielo.br/scielo.php?script=sci_ arttext\&pid=S0034-71672013000200005

4. Sociedade Brasileira de Nefrologia. Censo de diálise 2013[internet]. 2013 [Cited 2015 Apr 15]. Available from: http://arquivos.sbn.org.br/pdf/ censo_2013_publico_leigo.pdf

5. Frazão CMFQ, Ramos VP, LIRA ALBC. Qualidade de vida de pacientes submetidos à hemodiálise. Rev Enferm UERJ[internet]. 2011[Cited 2015 Apr 5]; 19(4): 577-82. Available from: http://www. facenf.uerj.br/v19n4/v19n4a12.pdf

6. Machado EL, Cherchiglia ML, Acúrcio FA. Profile and clinical outcome of patients in waiting list for kidney transplantation, Belo Horizonte (MG, Brazil), 2000-2005. Ciência \& Saúde Coletiva[internet]. 2011[Cited 2015 Jan 8]; 16(3): 1981-92. Available from:http:// www.scielo.br/scielo.php? pid=S1413$-81232011000300032 \&$ script=sci_arttext

7. Peeters MJ, Van Zuilen AD; Van den Brand JA, Peeters MJ, Zuilen ADV, Brand JAJGVD, Michiel LB, Buren MV, Dam MAGJT, Kaasjager KAH, Ligtenberg G, Sijpkens YWJ, Sluiter HE, JGVV Peter, Vervoort G, Vleming LJ, Blankestijn PJ, Wetzels JFM. Nurse Practitioner Care Improves Renal Outcome in Patients with CKD. J Am Soc Nephrol[internet]. 2014[Cited 2015 Apr 5]; 25(2): 390-8. Available from:http://www.ncbi.nlm.nih.gov/pmc/articles/ PMC3904557/

8. Bordinhão RC, Almeida MA. Instrument to collect data for critical patients based on the theory of basic human needs. Rev. Gaúcha Enferm [internet]. 2012[Cited 2015 Apr 5]; 33(2): 125-31. Available from:http://www. scielo.br/scielo.php?script=sci_arttext\&pid =S1983-14472012000200018

9. Ortiz F, Aronen P, Koskinen PK, Malmstrom RK, Finne $\mathrm{P}$, Honkanen EO, et al. Health-related quality of life after kidney transplantation: who benefits the most? Transpl Int[internet]. 2014 [Cited 2016 May 14]; 27(11):1143-51. Available from: http:// www.ncbi.nlm.nih.gov/pubmed/24977951

10. Horta WA. Processo de Enfermagem. São Paulo: EPU; 1979.

11. Bastos MG, Kirsztajn GM. Chronic kidney disease: importance of early diagnosis, immediate referral and structured interdisciplinary approach to improve outcomes in patients not yet on dialysis. J Bras Nefrol[internet]. 2011[Cited 2015 Apr 5]; 33(1): 93-108. Available from:http:// www.scielo.br/scielo.php? pid=S0101$-28002011000100013 \&$ script=sci_arttext

12. Fernandes MICD, Medeiros ABA, Macedo BM, Vitorino ABF, Lopes MVO, Lira ALBC. Prevalence of nursing diagnosis of fluid volume excess in patients undergoing hemodialysis. Rev Esc Enferm USP[internet]. 2014[Cited 2015 Apr 5]; 48(3):446453. Available from:http://www.scielo.br/pdf/ reeusp/v48n3/0080-6234-reeusp-48-03-446.pdf.

13. Dallé J, Lucena AF. Nursing diagnoses identified in hospitalized patients during hemodialysis. Acta Paul Enferm[internet]. 2012[Cited 2015 Apr 15]; 25(4): 504-10. Available from:http:// www.scielo.br/scielo.php?script=sci_arttext\&pi $\mathrm{d}=$ S0103-21002012000400004

14. Legendre C, Canaud G, Martinez F. Factors influencing long-term outcome after kidney transplantation. Transplant Int[internet]. 2014[Cited 2016 May 14]; 27(1):19-27, Available from: http:// www.ncbi.nlm.nih.gov/pubmed/24138291

15. Suszynski TM, Gillingham KJ, Rizzari MD, DunnTB, Payne WD, Chinnakotla S, et al. Prospective Randomized Trial of Maintenance immunosuppression With Rapid Discontinuation of Prednisone in Adult Kidney Transplantation. American Journal of Transplantation[internet]. 2013[Cited 2015 Apr 5]; 13: 961-970. Available from:http://www.ncbi. nlm.nih.gov/pmc/articles/PMC3621067/

16. Sampaio RMM, Coelho MO, Pinto FJM, Osteme EPR. Perfil epidemiológico de pacientes nefropatas e as dificuldades no acesso ao tratamento. Rev Bras Promoç Saúde[internet]. 2013[Cited 2015 Apr 5]; 26(1): 95-101. Available from:http://ojs. unifor.br/index.php/RBPS/article/view/2635

17. Chisholm-Burns MA, Erickson SR, Spivey CA, Kaplan B. Health-related quality of life and employment among renal transplant recipients. 
Clin Transplant[internet]. 2012 [Cited 2016 May 14];26(3):411-7.Available from: http://www.ncbi. nlm.nih.gov/pubmed/22032257

18. Xia Y, Kellems RE. Angiotensin Receptor Agonistic Autoantibodies and Hypertension: Preeclampsia and Beyond. Circulation Research[internet]. 2013[Cited 2015 Apr 5]; 113(1): 78-87. Available from:http://www.ncbi.nlm.nih.gov/pmc/articles/ PMC4131731/

19. Bissonnette J, Woodend K, Davies B, Stacey D, Knoll GA. Evaluation of a collaborative chronic care approach to improve outcomes in kidney transplant recipients. Clin Transplant[internet]. 2013[Cited 2015 Apr 5]; 27(2): 232-8. Available from: http://www.ncbi.nlm.nih.gov/pubmed/23330974

20. Rossiter MW, Valença MP, Valois AA. Transplante Renal: conhecimento do paciente acerca do período perioperatório. J Bras Transpl[internet]. 2012[Cited 2015 Apr 5]; 15: 1620-1650. Available from: http://www.abto.org.br/abtov03/Upload/ file/JBT/2012/1.pdf
Todos os autores participaram das fases dessa publicação em uma ou mais etapas a seguir, de acordo com as recomendações do International Committe of Medical Journal Editors (ICMJE, 2013): (a) participação substancial na concepção ou confecção do manuscrito ou da coleta, análise ou interpretação dos dados; (b) elaboração do trabalho ou realização de revisão crítica do conteúdo intelectual; (c) aprovação da versão submetida. Todos os autores declaram para os devidos fins que são de suas responsabilidades o conteúdo relacionado a todos os aspectos do manuscrito submetido ao OBJN. Garantem que as questões relacionadas com a exatidão ou integridade de qualquer parte do artigo foram devidamente investigadas e resolvidas. Eximindo, portanto o OBJN de qualquer participação solidária em eventuais imbróglios sobre a materia em apreço. Todos os autores declaram que não possuem conflito de interesses, seja de ordem financeira ou de relacionamento, que influencie a redação e/ou interpretação dos achados. Essa declaração foi assinada digitalmente por todos os autores conforme recomendação do ICMJE, cujo modelo está disponível em http://www. objnursing.uff.br/normas/DUDE_final_13-06-2013.pdf

Recebido: 02/07/2015

Revisado: 20/05/2015

Aprovado: 21/05/2015 